\title{
Fatores Associados à Asfixia Perinatal
}

\author{
Factors Associated with Perinatal Asphyxia
}

Alfredo de Almeida Cunha, Daniel de Souza Fernandes, Paula Frade de Melo, Marcela Hottum Guedes

\section{RESUM0}

Objetivo: avaliar fatores de risco associados ao índice de Apgar baixo.

Métodos: o estudo teve delineamento transversal. A população de estudo foi amostra randômica da população internada em maternidade nível III no ano de 2001. O desfecho foi indice de Apgar baixo, definido como de 1-6 (grupo de estudo) comparado a 7-10 (controle) no primeiro minuto. A primeira etapa foi avaliar a associação isolada de cada possível fator de risco. A segunda etapa consistiu em análise multivariada com modelagem usando regressão logística (modo passo a passo, reverso).

Resultados: houve 39 (14\%) recém-nascidos (RN) deprimidos que foram comparados a 238 (86\%) não deprimidos. A análise final (multivariada) revelou associação do índice de Apgar baixo com os seguintes fatores de risco: antecedente de natimorto $(O R=52,6)$, ameaça de parto prematuro, caracterizada pela existência de contrações uterinas não típicas de trabatho de parto $(O R=33,8)$, baixo peso do $R N$, inferior a $2.500 \mathrm{~g}(O R=11,2)$, antecedente de cesariana $(O R=7,4)$. Funcionaram como fatores de proteção o peso do $R N$ medido em gramas $(O R=0,9)$, sexo feminino do $R N(O R=0,1)$, presença de intercorrência clínica $(O R=0,4) e$ prematuridade, com idade gestacional inferior a 37 semanas $(O R=0,1)$.

Conclusão: o estudo do resultado pode auxiliar na identificação de fetos com risco de asfixia, possibilitando seu encaminhamento dentro do sistema de saúde, bem como o planejamento da assistência em unidades terciárias.

PALAVRAS-CHAVE: Asfixia. Prematuridade. Restrição do crescimento intra-útero.

\section{Introdução}

O objetivo de toda gravidez é a obtenção de recém-nascido $(\mathrm{RN})$ sadio com mínimo trauma para a mãe. Algumas vezes isto não é conseguido devido a complicações durante a gravidez, o parto ou com o concepto. Todas essas circunstâncias podem estar interligadas, resultando em um RN deprimido (asfixia perinatal).

A asfixia fetal é condição caracterizada por acidemia metabólica ou mista (metabólico-respi-

Hospital Geral de Bonsucesso, Rio de Janeiro, RJ, Brasil Correspondência

Alfredo de Almeida Cunha

Av. João Carlos Machado 427, ap 102 - Barra da Tijuca

22620-081 - Rio de Janeiro - RJ

Telefone: (21) 2492-3766 - FAX: (21) 2492-3547

e-mail: aacunha@uol.com.br

O trabalho foi realizado com auxílio de bolsas do Programa de Iniciação Científica da Universidade Estácio de Sá ratória) com $\mathrm{pH}$ menor do que 7 , podendo estar associada a um indice de Apgar de 0 a 3 por mais de 5 minutos e seqüelas neurológicas neonatais, tais como convulsões, coma ou hipotonia ou falência múltipla de órgãos ${ }^{1}$.

A avaliação clínica do RN foi proposta por Virginia Apgar em 1953 e 1958 e foi muito útil no julgamento da necessidade da ressuscitação do mesmo quando aplicada com um minuto de vida e novamente com cinco minutos ${ }^{1}$. Pode ser a única forma de avaliação em países em desenvolvimento, onde os exames laboratoriais podem não estar disponiveis. O índice de Apgar baixo é útil para identificar as crianças que necessitam de cuidados adicionais, mesmo na ausência de dados laboratoriais.

Existe consenso de que um índice de Apgar de 7-10 significa um bebê sadio que provavelmente não terá problemas futuros ${ }^{2}$. O índice de Apgar inferior a 7 é sinal de alerta para atenção especial. Há diferentes níveis de índice de Apgar baixo, 
de acordo com a alteração fisiopatológica. Além disso, o índice é parcialmente dependente da maturidade do concepto. As condições maternas também podem influir no índice, tais como medicações. E, finalmente, as próprias condições do RN influem na avaliação como, por exemplo, malformações neuromusculares ou cerebrais e condições respiratórias.

A asfixia neonatal é causa importante de morbidade e mortalidade neonatal que pode ser evitada nos países em desenvolvimento, como pôde ser observado em programa de treinamento implantado em 1990 na Índia com declínio significativo dos óbitos relacionados à asfixia ${ }^{3}$.

Em outro estudo a asfixia esteve presente no período anteparto em $34 \%$ de partos prematuros. Os fatores preditivos que levaram à intervenção e diagnóstico incluíram fatores clínicos e alterações dos testes de avaliação fetal. O aumento da freqüência de asfixia moderada ou grave durante a gravidez interrompida prematuramente implica maior probabilidade de morbidade em longo prazo ou óbito ${ }^{4}$. A asfixia esteve ainda presente em metade dos óbitos neonatais e mais freqüente em RN com menos de $2.000 \mathrm{~g}^{5}$.

Oswyn et al. ${ }^{6}$ observaram que algumas causas de asfixia perinatal são inevitáveis, mas algumas outras podem ser evitadas prestando-se assistência à gravidez de alto risco em unidades com facilidades obstétricas e boa assistência neonatal. Tentando definir a pesquisa de prioridades para a redução da morbidade e mortalidade perinatal nos países em desenvolvimento, Moss et al. ${ }^{7}$ relatam que $98 \%$ de todos os óbitos neonatais ocorrem nestes países, a maioria por parto domiciliar relacionados a infecções, asfixia no parto, traumatismos e conseqüências de prematuridade, baixo peso e anomalias congênitas.

O objetivo do estudo é, portanto, identificar fatores de risco para índice de Apgar baixo entre nós e avaliar sua relação por meio da análise multivariada.

\section{Métodos}

O desenho do estudo foi de corte transversal. O tamanho amostral foi calculado para estimar prevalência presumida em $20 \%$, com erro tolerável de 0,05 e confiança de 95\%, sendo calculado em 245 casos.

Entretanto, a população internada na maternidade do Hospital Geral de Bonsucesso inclui pacientes que atenderiam o critério de exclusão: aquelas que necessitaram ou não de curetagem uterina por abortamento (espontâneo ou provocado), pacientes internadas por complicações obstétricas (prenhez ectópica, descolamento prematuro de placenta, sem concepto que pudesse ser incluído no estudo), intercorrências clínicas para tratamento e posterior acompanhamento ambulatorial (infecção urinária e outras). Assim, geramos amostra randômica (algoritmo do Stata), incluindo $20 \%(n=660)$ de 3300 internações no ano de 2001 em maternidade de nível III (Hospital Geral de Bonsucesso, subordinado ao Ministério da Saúde, Rio de Janeiro, RJ), admitindo que, após as exclusões, pudéssemos atingir o tamanho amostral calculado, o que se revelou adequado, com $277 \mathrm{RN}$.

O banco de dados foi construído com o auxílio do programa Sistema Informático Perinatal do Centro Latino-Americano de Perinatologia (SIP/CLAP) ${ }^{8}$, órgão da Organização Pan-Americana de Saúde/ Organização Mundial de Saúde (OPAS/OMS).

O critério de inclusão foi RN vivo com o mínimo de 20 semanas de idade gestacional, definida pela data da última menstruação e corrigida pelo exame ultra-sonográfico. O desfecho foi o índice de Apgar, definido como baixo quando de 1-6 (grupo de estudo), comparado ao grupo de 7-10 (controle). Aqueles com Apgar 0 foram excluídos para não haver confusão com óbito fetal ou neonatal.

Os fatores de risco para índice de Apgar baixo foram os constantes do SIP/CLAP ${ }^{8}$ e incluíram idade materna, fatores de ordem social (estado civil, grau de escolaridade e dúvida sobre a data da última menstruação), história obstétrica (gesta, para, abortos, cesáreas e número de RN vivos), assistência pré-natal (se presente e número de consultas), complicações clínicas e obstétricas (pré-eclâmpsia, um grupo incluindo qualquer tipo de doença materna, rotura prematura das membranas ovulares, ameaça de parto prematuro, sífilis, modo de parto, idade gestacional, peso e sexo do RN). A idade materna foi considerada como variável contínua e como variável dicotômica, com ponto de corte aos 35 anos. A assistência pré-natal foi considerada como presente quando o número de consultas foi superior a quatro. A ameaça de parto prematuro foi considerada quando da presença de contrações não típicas de trabalho de parto mas que eram motivo de queixa de dor por parte da paciente. A sífilis foi considerada presente quando a sorologia foi positiva. O grau de instrução foi dicotomizado em analfabetismo presente ou não, pelo risco de interferência na procura da assistência e possibilidade de cumpri-la quando presente. Em relação ao RN foi utilizada a dicotomia entre baixo peso presente, quando inferior a $2.500 \mathrm{~g}$, e ausente a partir deste. A idade 
gestacional foi dicotomizada em prematuridade, presente quando inferior a 37 semanas, e ausente a partir desta.

A comparação de proporções foi feita mediante a razão de prevalência e o teste $\chi^{2}$. A comparação de médias foi feita com o uso do teste $t$ de Student. A modelagem foi feita com o uso de regressão logística, no modo passo a passo, que possibilita avaliar o risco de cada fator controlado pelos demais, podendo haver mudança da associação observada na análise bivariada. A medida de efeito do fator de risco foi calculada pela razão de chances (OR), com seu respectivo intervalo de confiança e grau de significância de 0,05. A razão de chances superior a um indicou risco e menor, indicou proteção. A análise estatística foi feita com o programa estatístico Stata versão 6.

O projeto foi aprovado pelo Comitê de Ética em Pesquisa da Universidade Estácio de Sá.

\section{Resultados}

Foram selecionados para o estudo $277 \mathrm{RN}$, dos quais 14\% (39/277) com indice de Apgar baixo (1-6, grupo de estudo) e 86\% (238/277) com índice de Apgar de 7-10 (controle).

A Tabela 1 mostra a comparação de médias entre os grupos. A idade materna (menor no grupo de estudo), a idade gestacional (menor no grupo de estudo), o peso do RN (menor no grupo de estudo) e o antecedente de nascidos mortos (maior no grupo de estudo) foram significativos $(\mathrm{p}<0,05)$. A paridade, o número de abortos, cesáreas prévias, número de consultas pré-natais e número de RN vivos não foram significativos e indicam, em análise preliminar, maior risco na paciente mais jovem, na prematuridade, no menor peso e paciente com antecedente de natimortos.

A Tabela 2 mostra a comparação entre proporções, utilizando a razão de prevalência (RP). Aqui as variáveis foram analisadas como dicotômicas (presente ou ausente) para facilitar a interpretação clínica: idade materna (de 35 anos ou mais), abortos prévios e cesáreas prévias. Houve diferença significativa na presença de baixo peso do RN $(\mathrm{RP}=8,1)$, antecedente de natimorto $(\mathrm{RP}=6,1)$, primiparidade $(\mathrm{RP}=2,1)$, prematuridade $(\mathrm{RP}=2,4) \mathrm{e}$ ameaça de parto prematuro $(\mathrm{RP}=2,6)$.

Tabela 1 - Comparação de médias (variáveis contínuas)

\begin{tabular}{lccc}
\hline Variável & Controle & Estudo & $\mathbf{p}$ \\
\hline Idade gestacional (semanas) & $36,9 \pm 3,6$ & $33,8 \pm 6,3$ & $<0,001$ \\
Peso do RN (gramas) & $2905 \pm 664$ & $2000 \pm 885$ & $<0,001$ \\
Idade materna (anos) & $24,5 \pm 6,7$ & $21,8 \pm 6,3$ & 0,02 \\
Número de consultas no & $4,0 \pm 2,3$ & $3,7 \pm 1,9$ & 0,35 \\
pré-natal (n) & & & \\
\hline
\end{tabular}

Tabela 2 - Associação de asfixia com variáveis categóricas.

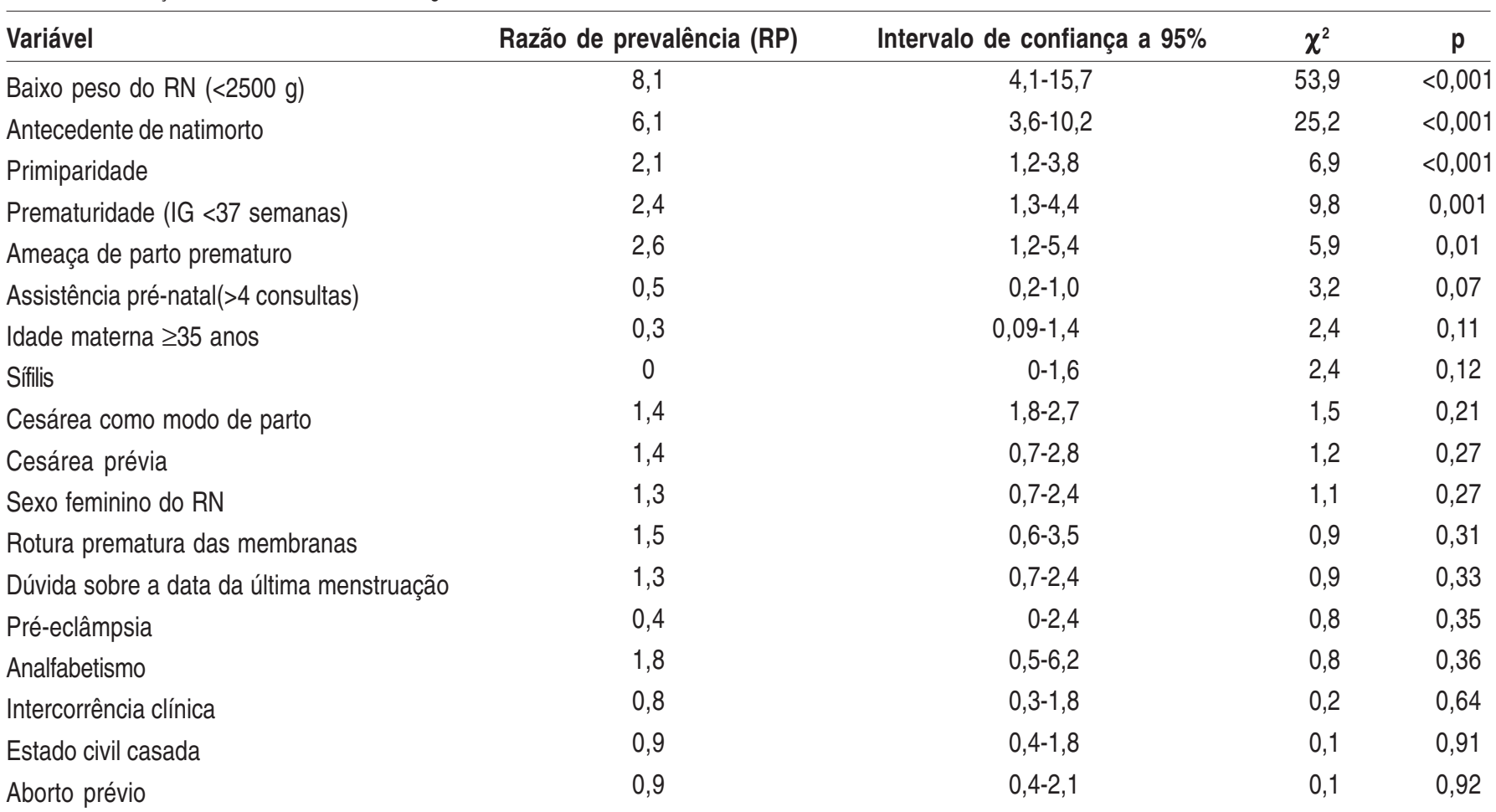

$\mathrm{IG}=$ idade gestacional. 
A Tabela 3 mostra o modelo pleno, inicialmente com todas as variáveis, sendo que só foram mantidas as variáveis com valor $\mathrm{p}<0,25$, pela técnica passo a passo de regressão logística. Utilizamos o critério de incluir todas as variáveis para não omitir nenhuma que pudesse ser significativa (reduz-se assim o erro beta). Foram considerados significativos os fatores com valor $p<0,05$. $O$ modelo inclui como fatores de risco o antecedente de natimorto $(\mathrm{OR}=52,6)$, a ameaça de parto prematuro $(\mathrm{OR}=33,8)$, o baixo peso do $\mathrm{RN}(\mathrm{OR}=11,2) \mathrm{e}$ $o$ antecedente de cesariana $(\mathrm{OR}=7,4)$. Foram fatores de proteção o peso do $\mathrm{RN}$ avaliado em gramas $(\mathrm{OR}=0,9)$, o sexo feminino do $\mathrm{RN}(\mathrm{OR}=0,1)$, a intercorrência clínica $(\mathrm{OR}=0,4)$ e a prematuridade $(\mathrm{OR}=0,1)$. Este modelo tem acurácia de $93 \%$, valor preditivo positivo de $83,3 \%$ e valor preditivo negativo de $94,3 \%$.

Tabela 3 - Modelo preditivo de asfixia neonatal.

\begin{tabular}{lcccc}
\hline Fator de risco & Razão de chances & Intervalo de confiança a 95\% & $\mathbf{z}$ & $\mathbf{p}$ \\
\hline Antecedente de natimorto & 52,6 & $4,0-675$ & 2,0 & 0,002 \\
Ameaça de parto prematuro & 33,8 & $1,6-695$ & 2,2 & 0,02 \\
Baixo peso do RN (<2500 g) & 11,2 & $1,3-92,4$ & 2,2 & 0,02 \\
Antecedente de cesariana & 7,4 & $1,8-30,2$ & 2,8 & 0,005 \\
Primiparidade & 6,9 & $1,6-29,2$ & 2,6 & 0,08 \\
Antecedente de aborto & 3,5 & $0,7-17,1$ & 1,5 & 0,12 \\
Número de consultas pré-natais & 1,7 & $1,0-3,0$ & 2,0 & 0,04 \\
Peso do RN (gramas) & 0,9 & $0,9-0,9$ & $-3,4$ & 0,001 \\
Sexo feminino & 0,1 & $0,1-0,7$ & $-2,3$ & 0,01 \\
Intercorrência clínica & 0,4 & $0-0,7$ & $-2,1$ & 0,02 \\
Prematuridade & 0,1 & $0,1-0,8$ & $-2,1$ & 0,03 \\
Assistência pré-natal (>4 consultas) & 0,1 & $0,1-2,7$ & $-1,2$ & 0,20 \\
\hline
\end{tabular}

Razão de prevalência $\chi^{2}=101,77 \quad p<0,001$; Acurácia $=93 \%$; Área sob a curva $=0,91$

Sensibilidade $=64,10 \%$

Valor preditivo positivo $=83,33 \%$

Especificidade $=97,90 \%$

Valor preditivo negativo $=94,33 \%$

\section{Discussão}

O primeiro dado demográfico avaliado foi a idade materna, menor no grupo de estudo na análise inferencial, mas que não se mantém significativa na modelagem, o que concorda com Adesina e Olayemi ${ }^{9}$, que não a identificaram como fator de risco, mesmo na presença de macrossomia.

A história obstétrica é referida como fator de risco quando considerados os abortos prévios ${ }^{10}$, com razão de chances de 2,6 , não confirmada pela nossa observação, em que apesar da razão de prevalência de 3,5 não foi significativa $(p<0,12)$. Ainda quanto aos antecedentes, a natimortalidade e a neomortalidade foram valorizadas como fatores de risco para asfixia perinatal ${ }^{6}$, com o que concordamos, uma vez que o antecedente de natimorto foi altamente significativo, com razão de chances de 52,6.

No grupo de fatores de ordem social, o estado civil solteira foi observado como fator de risco, o que não foi observado no presente estudo, em que o estado civil foi eliminado no processo de modelagem.

Em outro modelo, Milson et al. ${ }^{11}$ valorizaram os parâmetros cardiotocográficos, a eliminação intra-uterina de mecônio, o parto operatório, parto pélvico, o estado civil solteira, uso de ocitocina, complicações do cordão umbilical e compressão externa no auxílio ao parto. Destes, o antecedente de cesariana foi por nós observado como significativo, talvez em decorrência dos demais fatores ligados ao parto.

O perfil da paciente foi estudado quanto à primiparidade, chegando a ser valorizada como fator de risco ${ }^{12}$, o que não confirmamos, uma vez que a primiparidade não se mostrou significativa no processo de modelagem, o que a considerou controlada pelos demais fatores.

A indução é procedimento útil para a antecipação do parto, mas encontrou-se associação com indice de Apgar baixo ${ }^{13}$. O valor do trabalho de parto longo sobre o bem-estar fetal tem sido objeto de controvérsia. Alguns estudos tentam associar as variáveis do equilíbrio ácido-básico à duração do pri- 
meiro e do segundo períodos do trabalho de parto. Hagelin e Leyon ${ }^{14}$, por exemplo, não encontraram correlação entre a duração do primeiro período e $\mathrm{pH}$ ou excesso de bases da artéria umbilical. Entretanto, encontraram correlação entre a duração do período expulsivo e a diferença de $\mathrm{pH}$ e excesso de bases na artéria umbilical, mas a duração do período expulsivo por três horas causou diferença de apenas 0,05 no pH e diferença de excesso de bases de apenas $2,1 \mathrm{mmol} / \mathrm{L}$. Este efeito é tão pequeno que é difícil valorizá-lo clinicamente. Apesar desta observação, outros consideram que a atenção na condução do trabalho de parto pode reduzir a mortalidade perinatal pela redução da asfixia ${ }^{15,16}$. A asfixia intraparto pode ser prevenida por procedimento padrão (ausculta fetal a cada 30 minutos durante o primeiro período e a cada 5 minutos durante o período expulsivo).

$\mathrm{O}$ sexo masculino do $\mathrm{RN}$ foi associado ao aumento do risco de sofrimento fetal em estudo no qual foram incluídas 423.033 gestações únicas do Registro Nacional Holandês ${ }^{17}$, com o que concorda o presente estudo, uma vez que foi observado efeito protetor do sexo feminino.

A prática da cesariana, ao contrário de ser protetora do índice de Apgar baixo, pode ser fator de risco, quando o número de cesáreas ultrapassava o número predito de cesáreas ${ }^{18}$. Observamos que a cesárea não funcionou como fator de risco para índice de Apgar baixo, tendo sido excluída do modelo multivariado. Devemos lembrar que esta visão (de que cesariana é fator de risco para asfixia) apresenta um viés, uma vez que grande parte das operações cesarianas já tem como indicações o comprometimento fetal ou parto prematuro, eventualmente iatrogênico, na presença de sofrimento fetal crônico que obriga à antecipação do parto, ou seja, a asfixia é prévia à cesariana e não por ela determinada. Na verdade a operação é feita como forma de tratamento da asfixia.

Sutton et al. ${ }^{19}$ identificaram em análise multivariada associação de asfixia com primigrávida, doença da tiróide, qualquer complicação antenatal, crescimento intra-uterino restrito, sexo masculino, idade gestacional, rotura das membranas ovulares prolongada, complicações do trabalho de parto, indução do trabalho de parto, hipertermia materna, hemorragia de origem placentária durante o trabalho de parto, parto a fórceps e cesárea de emergência.

Com o uso do modelo logístico, Heinonen e Saarikoski ${ }^{12}$ identificaram associação de asfixia fetal com descolamento prematuro de placenta, primiparidade, alcoolismo, baixo peso ao nascer, pré-eclampsia, sexo masculino e pequeno para a idade gestacional. Outro estudo ${ }^{20}$ comparou os re- sultados de dois momentos da análise estatística em estudo prospectivo de $969 \mathrm{RN}$ de portadoras de gravidez de alto risco por prazo de cinco anos (19931997) na Tailândia. Na análise inferencial houve associação de asfixia perinatal com peso ao nascer, idade gestacional, prematuridade e parto pélvico. Entretanto, a análise multivariada com modelo logístico passo a passo mostrou que somente o peso do RN estava associado com asfixia perinatal. Os autores sugerem que, em países onde os recursos sejam limitados, uma equipe de ressuscitação deveria estar disponível para assistência a partos de conceptos com baixo peso (o que está freqüentemente associado à prematuridade e à apresentação pélvica).

$\mathrm{Na}$ análise bivariada foram observados como fatores de risco para índice de Apgar baixo no primeiro minuto menor idade materna, menor idade gestacional, menor peso do RN, quando da comparação de médias, e baixo peso ao nascer $(<2.500$ $\mathrm{g}$ ), antecedente de natimortalidade, primiparidade, prematuridade (idade gestacional $<37$ semanas) e ameaça de parto prematuro, quando comparadas as proporções.

A análise multivariada com modelo logístico incluiu no início todas as variáveis (modo passo a passo) e podemos observar três grupos de variáveis, considerando o nível de significância do resultado estatístico em 0,05. O primeiro, de fatores de risco propriamente dito (com aumento da probabilidade de ocorrência de asfixia, merecendo encaminhamento para unidade especializada), incluiu o antecedente de natimorto, a ameaça de parto prematuro, o baixo peso ao nascer, o antecedente de cesariana e o número de consultas prénatais. O segundo grupo foi de fatores de proteção (que diminuem o risco de asfixia e identificados pelo valor $z$ negativo) e incluiu o peso do $\mathrm{RN}$ (em gramas), o sexo feminino do RN, a intercorrência clínica e a prematuridade. O terceiro grupo foi daqueles que, embora conservados no modelo (porque apresentaram $\mathrm{p}<0,25)$, não mostraram significância, a saber: a primiparidade, o antecedente de aborto e a assistência pré-natal. As demais variáveis foram excluídas do modelo por apresentarem $\mathrm{p}>0,25$.

O modelo merece algumas observações. A primiparidade, embora não tenha sido identificada como fator de risco com margem de erro de 0,05, apresentou margem de erro que não deve ser desprezada $(8 \%$ ou $\mathrm{p}=0,08)$. Talvez com tamanho amostral maior ela fosse significativa. O número de consultas foi observado como fator de risco. Entendemos que o maior número de consultas esteja associado à gravidez de alto risco, introduzindo um viés. Por outro lado, a assistência pré- 
natal (caracterizada por mais de quatro consultas) não se mostrou efetiva na redução do risco, talvez por duas razões: a primeira é de que a asfixia pode depender de fatores ligados ao parto e a segunda é que o serviço funciona como pronto socorro obstétrico, recebendo pacientes sem assistência prénatal, introduzindo um outro tipo de viés, o de seleção. A prematuridade foi observada como fator de proteção porque, analisada em conjunto com o baixo peso, teve maior importância na predição do risco. A intercorrência clínica foi observada como fator de proteção para asfixia e interpretamos este achado como condução satisfatória da mesma durante a gravidez.

Apesar das limitações, o modelo demonstrou boa acurácia (93\%), alta especificidade (97\%) e alto valor preditivo negativo (94\%), o que permite excluir o risco com boa margem de segurança.

Concluímos que o modelo pode ajudar na predição do risco e orientar quanto a duas providências: fluxo da paciente no sistema de saúde e perfil da unidade onde deve ser assistida. As pacientes portadoras dos fatores de risco devem ser encaminhadas dentro do sistema de saúde para unidades onde possam receber assistência de acordo com o risco (unidade intensiva neonatal na presença dos fatores de risco e maternidade especializada no acompanhamento de gravidez de alto risco) e encaminhando partos de baixo risco para outras unidades. O planejamento da assistência pode beneficiar-se com a previsão do risco e a providência de equipe especializada em ressuscitação neonatal.

\section{ABSTRACT}

Purpose: to assess risk factors for low Apgar score. Methods: this was a cross-sectional study preformed in a random sample of patients admitted to a level III maternity hospital in 2001. The outcome was low Apgar score defined as an Apgar score 1-6 (study group) versus Apgar score 710 (control group) in the first minute of life. The first step was the evaluation of the association of each possible risk factor with low Apgar score. The second step was multivariate analysis with the backward stepwise logistic regression model.

Results: there were 39(14\%) depressed newborns which were compared to $238(86 \%)$ not depressed babies. The final analysis (multivariate) showed an association between low Apgar score and previous case of stillbirth $(O R=52.6)$, preterm labor threat $(O R=33.8)$, low birth weight, less than $2,500 \mathrm{~g}$ body weight $(O R=11.2)$ and previous cesarean section $(O R=7.4)$. Some factors acted as a protection, including birth weight, in grams $(O R=0.9)$, female sex of the newborn $(O R=0.1)$, medical complications $(O R=0.4)$ and prematurity (gestational age $<37$ weeks, $O R=0.1$ ). Conclusion: the study may help in the identification offetuses at great risk of asphyxia, allowing proper reference within the health system and planning of effective assistance in neonatal intensive care units.

KEYWORDS: Asphyxia. Prematurity. Intrauterine growth restriction.

\section{Referências}

1. Cunningham FG, Gant NF, Leveno KJ, Gilstrap LC $3^{\text {rd }}$, Hauth JC, Wenstrom KD. The newborn infant. In: Williams Obstetrics. $21^{\text {st }}$ ed. New York: McGrawHill; 2001. p.385-402.

2. Casey BM, McIntire DD, Leveno KJ. The continuing value of the Apgar score for the assessment of newborn infants. N Engl J Med 2001; 344:467-71.

3. Deorari AK, Paul VK, Singh M, Vidyasagar D. The National Movement of Neonatal Resuscitation in India. J Trop Pediatr 2000; 46:315-7.

4. Low JA, Pickersgill H, Kilten H, Derrick EJ. The prediction and prevention of intrapartum asphyxia in term pregnancies. Am J Obstet Gynecol 2001; 184:724-30.

5. Tripathy R, Parida SN, Tripathy SN, Devi PS, Das RN, Swain A. Physical status of newborns and neonatal outcome. Indian J Pediatr 2002; 69:1401-5.

6. Oswyn G, Vince JD, Friesen H. Perinatal asphyxia at Port Moresby General Hospital: a study of incidence, risk factors and outcome. P N G Med J 2000; 43:110-20.

7. Moss W, Darmstadt GL, Marsh DR, Black RE, Santosham M. Research priorities for the reduction of perinatal and neonatal morbidity and mortality in developing country communities. J Perinatol 2002; 22:484-95.

8. Simini F, Rubino M, López R, Diaz AG, Schvarcz R. Procesamiento de datos del sistema informático perinatal. $2^{\text {a }}$ ed. Montevideo: Centro Latinoamericano de Perinatologia y Desarrollo Humano; 1996. (Publ. Científica, 1362).

9. Adesina AO, Olayemi O. Fetal macrosomia at the University College Hospital, Ibadan: a 3-year review. J Obstet Gynaecol 2003; 23:30-3.

10.Suka M, Sugimori H, Nakamura M, Haginiwa, Yoshida K. Risk factors of low Apgar score in Japanese full-term deliveries: a case-control study. J Epidemiol 2002; 12:320-3.

11.Milson I, Ladfors L, Thiringer K, Niklasson A, Odeback A, Thornberg E. Influence of maternal, 
obstetric and fetal risk factors on the prevalence of birth asphyxia at term in a Swedish urban population. Acta Obstet Gynecol Scand 2002; 81:909-17.

12.Heinonen S, Saarikoski S. Reproductive risk factors of fetal asphyxia at delivery: a population based analysis. J Clin Epidemiol 2001; 54:407-10.

13.Duff C, Sinclair M. Exploring the risks associated with induction of labour: a retrospective study using the NIMATS database. Northern Ireland Maternity System. J Adv Nurs 2000; 31:410-7.

14.Hagelin A, Leyon J. The effect of labor on the acidbase status of the newborn. Acta Obstet Gynecol Scand 1998; 77:841-4.

15.Buchmann EJ, Pattinson RC, Nyathikazi N. Intrapartum-related asphyxia in South Africa- lessons from the first national perinatal care survey. S Afr Med J 2002; 92:897-901.

16.Pattinson RC. Challenges in saving babies - avoidable factors, missed opportunities and substandard care in perinatal deaths in South Africa. S Afr Med J 2003; 93:450-5.
17.Bekedam DJ, Engelsbel S, Mol BW, Buitendijk SE, van der Pal-de-Bruin KM. Male predominance in fetal distress during labor. Am J Obstet Gynecol 2002; 187:1605-7.

18. Bailit JL, Garrett JM, Miller WC, McMahon MJ, Cefalo RC. Hospital primary cesarean delivery rates and the risk of poor neonatal outcomes. Am J Obstet Gynecol 2002; 187:721-7.

19.Sutton L, Sayer GP, Bajuk B, Richardson V, Berry G, Henderson-Smart DJ. Do very sick neonates born at term have antenatal risks? 1. Infants ventilated primarily for problems of adaptation to extra-uterine life. Acta Obstet Gynecol Scand 2001; 80:905-16.

20.Kolatat T, Vanprapar N, Thitadilok W. Perinatal asphyxia: multivariate analysis of risk factors. J Med Assoc Thai 2000; 83:1039-44.

Recebido em: 19/7/2004 Aceito com modificações em: 12/11/2004 